\title{
Évolution lointaine inattendue d'une contamination interne
}

\author{
L. ANDRIEU et M. FATOME (*)
}

(Manuscrit reçu le 23 mars 1979)

\begin{abstract}
RÉSUMÉ
Des données récentes montrent qu'après une contamination respiratoire par ${ }^{137} \mathrm{Cs}$ et ${ }^{60} \mathrm{Co}$, une faible fraction de la charge corporelle s'élimine avec une période très longue. Il n'est pas possible d'en préciser actuellement la cinétique. Cette constatation prouve que le modèle de MorRow et les équations de rétention proposées par la C.I.P.R. [11] ne décrivent pas la cinétique dans toute sa complexité
\end{abstract}

\begin{abstract}
New data indicate that, after a contamination by inhalation with ${ }^{137} \mathrm{Cs}$ and ${ }^{60} \mathrm{Co}$, a small fraction of the body burden is eliminated with a very long period. At the present time, it is not possible to specify the kinetics. These findings prove that the lung deposition and retention model and retention equations proposed by I.C.R.P. [11] cannot describe the kinetics in their whole complexity.
\end{abstract}

\section{INTRODUCTION}

Le 5 décembre 1970, un volontaire réalise sur lui-même une contamination interne expérimentale par voie respiratoire. Les paramètres qualificatifs et quantitatifs de l'expérience sont préalablement mesurés avec le maximum de soin de telle sorte que la dose engagée reste inférieure à la dose maximale admissible. De très nombreuses mesures de spectrométrie gamma et de radiochimie des excréta ont permis de suivre cette contamination. Les mesures faites dans nos laboratoires ont duré 1 an. La spectrométrie gamma a été faite également par des laboratoires extérieurs aux nôtres et poursuivie pendant 6 ans. Les résultats ont fait l'objet de plusieurs publications [3, 4, 7]. En octobre 1977 est pratiqué un nouvel examen spectrométrique. Nous avons la

(*) Centre de Recherches du Service de Santé des Armées, Division d'Hygiène Atomique, 1 bis, rue du Lieutenant-Batany, 92141 Clamart. 
surprise de trouver des résultats que notre précédent bilan ne permettait pas de prévoir $[1,7]$. A la lumière de ces résultats, nous proposons une discussion de fond sur le modèle de Morrow et les équations de rétention proposées par la C.I.P.R.

\section{DÉROULEMENT DE L'OPÉRATION}

$1^{\circ}$ Quatre radionucléides prédominaient dans le mélange respiré avec le pourcentage indiqué par le tableau I. L'activité globale inhalée était de 1,6 $\mu \mathrm{Ci}$.

\section{TABLEAU I}

COMPOSITION DU MÉLANGE INHALÉ

\begin{tabular}{|c|c|}
\hline${ }^{137} \mathrm{Cs}$ & 15 p. cent \\
\hline${ }^{60} \mathrm{Co}$ & 52 p. cent \\
\hline${ }^{144} \mathrm{Ce}$ & 21 p. cent \\
\hline${ }^{90} \mathrm{Sr}$ & 0,4 p. cent \\
\hline
\end{tabular}

Seuls les éléments prédominants sont mentionnés.

$2^{\circ} \mathrm{Au}$ cours du bilan initial, on s'est proposé de voir si la fonction d'entrée donnée par le modèle de Morrow et la fonction de rétention donnée par la C.I.P.R. étaient vérifiées, ceci en étudiant la convergence de trois séries de données expérimentales :

(a) tous les paramètres respiratoires qui conditionnent la contamination;

(b) les mesures sur les excréta;

(c) les mesures de spectrométrie gamma sur l'organisme.

Les paramètres à vérifier ou à déterminer étaient au nombre de trois :

(a) le nombre de fractions qui suivent des cinétiques différentes;

(b) le poucentage de ces fractions;

(c) leur cinétique. Le bilan pratiqué en 1973 avait conduit à une légère modification de l'équation de rétention du césium 137 et avait conclu au fait qu'une fraction du dépôt alvéolaire du cobalt 60 avait une période de transfert de 400 jours.

$3^{\circ}$ Deux nouvelles spectrométries sont pratiquées les 10 et 14 octobre 1977. Nous avons la surprise de constater que 7 ans après la contamination, le sujet présente encore du ${ }^{60} \mathrm{Co}$ et du ${ }^{137} \mathrm{Cs}$ en quantité non négligeable. 
Il nous confirme qu'il subit régulièrement un contrôle spectrométrique qui a toujours montré la présence de ces deux radionucléides. Par ailleurs, le pic de ${ }^{137} \mathrm{Cs}$ dû aux retombées et constaté au niveau de la population a disparu depuis plusieurs années. Ces résultats ne sont pas conformes aux données de la littérature, tout au moins pour ce qui concerne ${ }^{137} \mathrm{Cs}[9,10]$. Pour ${ }^{60} \mathrm{Co}$, Newton et RUNDO ont trouvé des périodes longues de plusieurs années chez 5 individus à la suite d'une contamination accidentelle [6].

Pour préciser les mesures, nous faisons l'hypothèse vraisemblable mais discutable que ces radionucléides sont retenus au niveau des poumons et nous réalisons un mannequin étalon Remcal qui contient des charges connues de ${ }^{60} \mathrm{Co}$ et ${ }^{137} \mathrm{Cs}$ localisées dans les poumons (respectivement 35 et $5 \mathrm{nCi}$ ). Les deux examens donnent des spectres comparables.

\section{LE PROBLÈME DES MESURES EN SPECTROMÉTRIE GAMMA HUMAINE}

Cette expérimentation a permis de comparer les résultats de plusieurs installations de spectrométrie humaine et de voir combien ils peuvent être divergents. Ceci met en évidence les difficultés de la méthode, son manque de précision. En effet, la géométrie du comptage est liée à la morphologie du sujet et à la répartition du radionucléide mesuré. Or, l'étalonnage par mannequin implique le choix d'une morphologie et d'une répartition métabolique qui ne sont pas forcément celles des mesures réelles. De plus, lorsque la contamination est récente, cette répartition est rapidement changeante [5].

Le tableau II donne les résultats obtenus jusqu'au $1641^{\mathrm{e}}$ jour. Les mesures sont faites dans les conditions les plus reproductibles possibles [3].

Pour étudier la cinétique de la rétention des radionucléides, il faudrait tracer les courbes, et chercher une décomposition en courbes élémentaires. Cela suppose que les dernières mesures concernent seulement la fraction qui a l'élimination la plus lente, les autres fractions ayant disparu. Ce n'est que dans ce cas que l'on peut calculer une période à partir de deux mesures consécutives. Les valeurs calculées dans divers intervalles doivent être les mêmes. S'il n'en est pas ainsi, nous ne sommes pas en présence d'une exponentielle unique.

A titre indicatif, ce calcul a été fait dans tous les intervalles (colonnes 3 et 5).

\section{ÉVOLUTION DU CÉSIUM 137}

\section{1. Évaluation du dÉPÔT A L'ORIGINE}

Le dépôt pulmonaire à l'origine mesuré par spectrométrie est estimé à $200 \mathrm{nCi}$. Parallèlement, un calcul pratiqué à partir des paramètres respiratoires a donné $204 \mathrm{nCi}$. La concordance paraît excellente, mais les mesures de spectrométrie gamma rassemblées dans le tableau II, sont nettement supérieures. 
TABLEAU II

Charges Corporelles en ${ }^{137} \mathrm{Cs}$ et ${ }^{60} \mathrm{Co}$ Mesurées Par SPeCtrométrie gamma, D'APrès [3].

\begin{tabular}{|c|c|c|c|c|}
\hline $\begin{array}{l}\text { Temps écoulé depuis } \\
\text { la contamination }\end{array}$ & $\begin{array}{l}{ }^{137} \mathrm{Cs} \\
(\mathrm{nCi})\end{array}$ & $\begin{array}{l}\mathrm{T}_{e} \\
\text { (j) }\end{array}$ & $\begin{array}{l}{ }^{60} \mathrm{Co} \\
(\mathrm{nCi})\end{array}$ & $\begin{array}{l}\mathrm{T}_{e} \\
(\mathrm{j})\end{array}$ \\
\hline $1 \mathrm{~h} . \ldots \ldots \ldots \ldots$ & 250 & - & 890 & - \\
\hline $2 \mathrm{~h} . \ldots \ldots \ldots \ldots$ & 230 & - & 700 & 一 \\
\hline $3 \mathrm{~h} . \ldots \ldots \ldots \ldots$ & 210 & - & 600 & 一 \\
\hline $2 \mathrm{j} \ldots \ldots \ldots \ldots$ & 190 & 一 & 420 & - \\
\hline $7 \mathrm{j} \ldots \ldots \ldots \ldots$ & 170 & - & 300 & - \\
\hline $70 \mathrm{j} \ldots \ldots \ldots \ldots$ & 100 & 130 & 250 & - \\
\hline $105 \mathrm{j} \ldots \ldots \ldots \ldots$ & 83 & \multirow{2}{*}{108} & 190 & \multirow{2}{*}{230} \\
\hline $231 \mathrm{j} \ldots \ldots \ldots \ldots$ & 37 & & 130 & \\
\hline $378 \mathrm{j} \ldots \ldots \ldots \ldots$ & 14 & 104 & 100 & 388 \\
\hline $569 \mathrm{j}$ & 10 & 393 & 73 & 420 \\
\hline & & 706 & & 818 \\
\hline $1040 \mathrm{j} \ldots \ldots \ldots \ldots$ & 6,3 & 1160 & 49 & 847 \\
\hline $1641 \mathrm{j} \ldots \ldots \ldots \ldots$ & 4,4 & - & 30 & - \\
\hline
\end{tabular}

Dans les $3^{\mathrm{e}}$ et $5^{\mathrm{e}}$ colonne $\mathrm{T}_{e}$ représente la période effective calculée dans chaque intervalle entre deux mesures avec l'hypothèse qu'il ne reste qu'une seule fraction exponentielle lente. Les chiffres très dispersés montrent que cette hypothèse ne peut être retenue.

\section{CinÉTIQUe}

D'après le modèle de MorRow, le transfert du poumon vers le sang est rapide, le césium est dans la classe $D$ [11].

La C.I.P.R. donne une fonction de rétention à deux composantes exponentielles, l'une rapide (1 jour de période) et l'autre lente (110 jours) [2] :

$$
R(t)=0,15 e^{-(0,693 / 1) t}+0,85 e^{-(0,693 / 110) t} \quad(t \text { en jours })
$$


Les mesures faites au cours du premier bilan ont donc été interprétées sur la base d'une rétention à deux composantes et l'équation obtenue est un peu différente

$$
R(t)=0,5 e^{-(0,693 / 1,6) t}+0,5 e^{-(0,693 / 133) t} .
$$

Il y a donc une bonne concordance des périodes mais une nette différence en ce qui concerne les fractions.

Si l'on se réfère au modèle de Morrow, qui classe toujours le césium en classe $D$, et à l'équation de rétention à deux périodes proposée par la C.I.P.R. ou à celle calculée précédemment, la charge actuelle devrait être nulle. Dans le cas présent, il existe donc une faible fraction, évaluée à environ $5 \mathrm{nCi}$, qui s'élimine très lentement et pour laquelle il n'est pas possible, actuellement tout au moins, de définir une période.

\section{INTERPRÉTATION}

L'explication de ce phénomène n'est pas simple. Deux processus distincts se succèdent dans une contamination pulmonaire : l'épuration pulmonaire (modèle de MORRow); la rétention au niveau de l'organisme. de ${ }^{137} \mathrm{Cs}$ ?

Lequel de ces deux processus est responsable de la parsistance

(a) La rétention a fait l'objet de nombreux travaux qui présentent incontestablement une bonne cohérence. Ces travaux sont faits sur des animaux, mais peut-on en extrapoler les résultats à l'homme?

On ne connaît pas de rôle biologique du césium mais, chimiquement, il est semblable au potassium qui est intra-cellulaire, présent dans tout l'organisme. Il n'est pas impossible qu'une faible quantité de césium reste solidement piégée dans l'os.

(b) Le modèle de Morrow place le césium dans la classe $D$ (c'est-à-dire à épuration rapide), quelle que soit la forme chimique. Peut-être le mélange inhalé contenait-il du ${ }^{137} \mathrm{Cs}$ sous plusieurs formes? Peut-on être absolument certain que toutes ces formes chimiques sont facilement transférables?

Il n'est pas impossible que l'épuration pulmonaire soit responsable de la présence actuelle de ${ }^{137} \mathrm{Cs}$. A quel niveau serait-il piégé? On ne peut faire que des hypothèses. Le rhinopharynx et la trachée semblent devoir être éliminés. Il resterait donc les alvéoles pulmonaires et, plus vraisemblablement, les ganglions. Pour Morrow, les éléments classés en Y, contenus dans les ganglions pulmonaires gagnent le sang mais seulement dans la proportion de $1 / 10$, ce qui implique que $9 / 10$ restent sur place. Peut-être en est-il ainsi pour une faible quantité de ${ }^{137} \mathrm{Cs}$ ?

En somme, le classement proposé par Morrow en $D, W$ et $Y$, selon la vitesse d'épuration pulmonaire, paraît trop rigoureux pour rendre compte exactement de la réalité.

vol. $14-\mathrm{N}^{\circ} 3$ 


\section{ÉVOLUTION DU COBALT 60}

\section{1. Évaluation de la Charge a L'ORigine}

1 heure après la contamination, la première mesure de spectrométrie gamma portée dans le tableau II donne $890 \mathrm{nCi}$. Le calcul fait à partir des données de l'exposition donne $707 \mathrm{nCi}$, tandis que l'évaluation faite à partir des selles des premiers jours donne $950 \mathrm{nCi}$.

\section{Cinétique D'élimination}

Le modèle de Morrow classe les sulfures, sulfates, phosphates et carbonates de cobalt en classe $D$. Par contre, les carbures, oxydes, hydroxydes, halogénures et nitrates sont en classe $W$. Aucun composé connu n'est classé en $Y$. Mais avons-nous assez de données pour affirmer qu'il n'en existe pas?

En ce qui concerne la rétention, la C.I.P.R. propose une équation à quatre cinétiques

$$
\begin{aligned}
R(t)= & 0,54 e^{-(0,693 / 0,26) t}+0,32 e^{-(0,693 / 1,8) t} \\
& +0,086 e^{-(0,693 / 14) t}+0,054 e^{-(0,693 / 145) t}, \quad(t \text { en jours }),
\end{aligned}
$$

Cette équation est établie après injection intra-veineuse sur le chien suivi pendant plus de 400 jours, en spectrométrie gamma. On peut faire deux remarques :

(a) La forme sous laquelle est injecté le cobalt, n'est pas précisée.

(b) Les mesures de spectrométrie sont faites pendant 400 jours. Or, cela n'est pas suffisant pour mettre en évidence une éventuelle rétention beaucoup plus longue. Si cette rétention existe, le fait de l'ignorer perturbe les calculs surtout au niveau des deux dernières fractions qui sont très faibles $(0,086$ et 0,054 du total). Il y a donc une incertitude sur l'existence, la valeur et la période des fractions les plus lentes. Manifestement, la C.I.P.R. ne peut faire mieux que citer, à titre indicatif, les données de la littérature qui paraissent les plus intéressantes.

Si la période la plus longue était réellement de 400 jours, comme cela a été calculé au cours du bilan précédent, il ne resterait plus rien de mesurable en octobre 1977. La persistance de $35 \mathrm{nCi} \mathrm{de}{ }^{60} \mathrm{Co}$ prouve incontestablement qu'une faible fraction de la charge (environ $3 \mathrm{p}$. cent) s'élimine avec une très longue période. Les deux derniers chiffres calculés dans le tableau, donnent 818 jours et 847 jours, mais ce n'est pas suffisant pour affirmer qu'il s'agit bien de la période effective la plus longue.

\section{INTERPRÉTATION}

S'agit-il d'une rétention au niveau du poumon ou au niveau d'un autre organe? Autrement dit, faut-il incriminer le modèle d'épuration pulmonaire de Morrow ou la fonction de rétention? 
Pour la C.I.P.R., les travaux publiés suggèrent qu'une très faible fraction $\mathrm{du}{ }^{60} \mathrm{Co}$ est retenue avec une très longue période mais ne permettant pas de préciser davantage. Peut-être les temps d'observation n'ont-ils pas été assez longs pour autoriser une telle détermination. Cela donne toute sa valeur à la présente expérience.

On ne peut pas éliminer la possibilité d'une rétention au niveau des ganglions pulmonaires et si tel était le cas, le classement des éléments, tel que le propose MorRow, ne rendrait pas compte exactement des réalités.

\section{NOUVELLES DONNÉES}

K. V. RAGHAVENDRAN et al. ont relaté, récemment la contamination de travailleurs par l'iode 131, le césium 137 et le cobalt 60 [8]. En ce qui concerne l'iode 131 et le césium 137, l'évolution n'est pas en désaccord avec les équations proposées par la C.I.P.R. On ne constate pas la persistance à long terme d'une fraction de césium. Par contre, quatre de ces agents contaminés par ${ }^{60} \mathrm{Co}$ présentent une rétention à long terme d'une faible fraction de cobalt. Les auteurs tentent de calculer une période biologique pour cette fraction mais obtiennent des valeurs trop dispersées. La dispersion des résultats permet de mettre en doute la notion de période biologique. $\mathrm{Si}$, toutefois, la cinétique est vraiment exponentielle pour cette fraction de cobalt, la période d'élimination est très variable d'un sujet à l'autre et l'intérêt pratique de la notion de période s'en trouve très diminué. La valeur la plus élevée ( $25243 \mathrm{j}$.) est calculée sur une période d'observation de 1250 jours, qui paraît bien courte pour affirmer une telle rétention. Cette critique reste, à un moindre degré, valable pour les trois autres cas.

Nous retiendrons seulement la persistance à très long terme d'une faible fraction de ${ }^{60} \mathrm{Co}$ et sur ce point, il y a concordance avec notre observation.

\section{CONCLUSION}

Cette expérience unique est très riche d'enseignements les plus divers :

\section{Critique des modèles}

Les cinétiques invoquées reposent sur les notions classiques de " compartiments ". Or, les échanges entre ces compartiments sont supposés obéir à des cinétiques du $1^{\text {er }}$ ordre à coefficients constants, et chaque échange élémentaire d'un système de compartiments est exprimé par une expression de la forme

$$
d x=K x d t .
$$

On aboutit ainsi, pour les compartiments, à un système d'équations différentielles du $1^{\text {er }}$ ordre à coefficients constants que l'on sait résoudre. Les solutions sont des sommes d'exponentielles. Ce modèle semble dans bien des cas exprimer la réalité. Mais en est-il toujours ainsi? 
Le devenir d'un radioélément dans l'organisme fait intervenir des réactions chimiques. Or, la cinétique chimique nous apprend que les réactions sont d'ordre $1,2,3,1 / 2,3 / 2$, etc., et l'on écrirait par exemple

$$
\begin{gathered}
d x=K x^{2} d t \text { pour l'ordre } 2 \\
d x=K x^{1 / 2} d t \text { pour l'ordre de } 1 / 2 \text {, etc. }
\end{gathered}
$$

Si un système d'équations différentielles contient un terme différent de l'ordre 1, les solutions ne sont plus des exponentielles. Il en est de même si les coefficients ne sont pas constants et rien ne garantit qu'ils le sont toujours. Dans ces conditions, il ne faut pas s'étonner si les résultats obtenus s'éloignent souvent des modèles proposés.

\section{CRitique des expérimentations}

Les modèles proposés par la C.I.P.R. sont suggérés par l'expérimentation animale. Mais cette expérimentation réalise des situations qui sont peut-être différentes de celle de notre sujet. En effet, le cas présent dure depuis plus de 7 ans et nous voyons que ce délai est insuffisant pour connaître l'évolution à long terme. Les expériences animales sont, en général, beaucoup plus courtes. Un délai aussi long imposerait aux chercheurs une très lourde contrainte et le choix d'un animal qui vive assez longtemps. L'extrapolation à l'homme ne serait pas facile car il est possible que les cinétiques changent avec l'âge, et les "tranches de vie " concernées ne sont peut-être pas physiologiquement comparables?

\section{Dose engagéE}

Cette rétention à long terme entraîne pour un organe que nous ne connaissons pas une dose chronique que nous ne pouvons calculer puisque nous ignorons la concentration et la localisation du radioélément.

\section{REMERCIEMENTS}

Nous tenons à remercier le $\mathrm{D}^{\mathrm{r}} \mathrm{M}$. DelPla pour sa précieuse coopération.

\section{BIBLIOGRAPHIE}

[1] Heidelberg, 11-16 may 1964, Assessment of radioactivity in man Vienne, A.I.E.A., 1964.

[2] Commission Internationale de Protection Radiologique (C.I.P.R.). L'évaluation des doses de rayonnement reçues par les tissus de l'organisme après contamination interne d'origine professionnelle. Publication C.I.P.R., 10 (traduction française), Saclay, C.E.A., 1978.

[3] Delpa M., Moroni J. P., Bournay P., Schaeffer R., Gaulard R., Rocquet G., Chinardet J. M., Letard H. Évaluation sur 6 ans d'une contamination radioactive humaine expérimentale. C.R. Acad. Sc., Paris, 1976, 283, série D, 1465-1468. 
[4] Fatome M., Frossard H., Gueguen J. P., Prat G., Quentric Y., Rocquet G. Calcul des charges et des doses délivrées à partir de résultats expérimentaux de radiochimie et de spectrométrie humaine dans un cas de contamination interne chez l'homme. Travaux scientifiques du Centre de Recherches du Service de Santé des Armées, 1973, 54-59.

[5] Meneely G. R.. Linde Sh. M., Éds. Radioactivity in man, 2nd symposium on whole body counting and effects of internal gamma ray-emitting radioisotopes, Chicago, 5-7 september 1962, Springfield, Ch. C. Thomas, 1965.

[6] Newton D., Rundo J. The long-term retention of inhaled cobalt 60. Health Phys., 1971, 21, 377-384.

[7] PrAT G. Modèle et expérimentation humaine concernant l'inhalation de radioéléments. Thèse de doctorat en biologie humaine, Bordeaux-II, juin 1976.

[8] Raghavendran K. V., Satbhai P. D., Abhyankar B., Unnikrishnan K., SOMASUNDARAM S., Long-term retention studies of ${ }^{131} \mathrm{I},{ }^{137} \mathrm{Cs}$ and ${ }^{60} \mathrm{Co}$ in Indian workers. Health Phys., 1978, 34 185-187.

[9] Rundo J. A survey of the metabolism of caesium in man. Brit. J. Radiol., 1964, 37, 434, 108-113.

[10] Rundo J. A case of accidental inhalation of irradiated uranium. Brit. J. Radiol., 1965, 38, $445,39-50$.

[11] I.C.R.P. Task group on Lung dynamics. Health Phys., 1966, 12, 173-187. 